\title{
Report of whole-brain radiation therapy in a patient with an implanted deep brain stimulator: important neurosurgical considerations and radiotherapy practice principles
}

\author{
Rupesh Kotecha, MD, ${ }^{1}$ Camille A. Berriochoa, MD, ${ }^{1}$ Erin S. Murphy, MD, ${ }^{1,2}$ \\ Andre G. Machado, MD, PhD, ${ }^{3-6}$ Samuel T. Chao, MD, ${ }^{1,2}$ John H. Suh, MD, ${ }^{1,2}$ and \\ Kevin L. Stephans, MD'
}

\begin{abstract}
'Department of Radiation Oncology, Taussig Cancer Institute, ${ }^{2}$ Rose Ella Burkhardt Brain Tumor and Neuro-Oncology Center, Cleveland Clinic, ${ }^{3}$ Center for Neurological Restoration, and Departments of ${ }^{4}$ Neurological Surgery, ${ }^{5}$ Neurosciences, and ${ }^{6}$ Biomedical Engineering, Cleveland Clinic, Cleveland, Ohio
\end{abstract}

\begin{abstract}
Patients with implanted neuromodulation devices present potential challenges for radiation therapy treatment planning and delivery. Although guidelines exist regarding the irradiation of cardiac pacemakers and defibrillators, fewer data and less clinical experience exist regarding the effects of radiation therapy on less frequently used devices, such as deep brain stimulators. A 79-year-old woman with a history of coarse tremors effectively managed with deep brain stimulation presented with multiple intracranial metastases from a newly diagnosed lung cancer and was referred for whole-brain radiation therapy. She was treated with a German helmet technique to a total dose of $30 \mathrm{~Gy}$ in 10 fractions using $6 \mathrm{MV}$ photons via opposed lateral fields with the neurostimulator turned off prior to delivery of each fraction. The patient tolerated the treatment well with no acute complications and no apparent change in the functionality of her neurostimulator device or effect on her underlying neuromuscular disorder.

This represents the first reported case of the safe delivery of whole-brain radiation therapy in a patient with an implanted neurostimulator device. In cases such as this, neurosurgeons and radiation oncologists should have discussions with patients about the risks of brain injury, device malfunction or failure of the device, and plans for rigorous testing of the device before and after radiation therapy.
\end{abstract}

http://thejns.org/doi/abs/10.3171/2015.2.JNS142951

KEY WORDS radiation therapy; deep brain stimulator; neurostimulator; ionizing radiation; whole-brain; brain metastasis; oncology; functional neurosurgery

$\Psi_{\text {inp }}$ LECTRONIC devices and implants can present challenges for radiation therapy delivery, especially when an implanted device is located in the field of radiation exposure. Cardiac pacemakers and defibrillators are the most common devices encountered in radiation therapy, often in patients who present with thoracic or breast malignancies. Clinical experience with similarly functioning neuromodulation devices such as cochlear implants and deep brain stimulators is, however, very limited. Moreover, although guidelines exist regarding the irradiation of pacemakers and defibrillators, patients with less frequently used implanted devices are approached on a case-by-case basis due to lack of experience-informed guidelines. ${ }^{8,14} \mathrm{In}$ the present case study, we describe a patient with a history of tremors previously treated with implantation of a deep brain stimulation (DBS) unit, who developed multiple brain metastases from lung cancer and was subsequently referred for whole-brain radiation therapy.

\section{Case Report}

History and Presentation

A 79-year-old woman with a medical history significant for coarse tremors, hypertension, and diabetes mellitus 
presented to her neurologist with worsening balance difficulties, increasing weakness, fatigue, and memory difficulties. Her tremors had been treated by means of DBS, and she had undergone placement of a DBS unit with electrodes positioned in the thalamus (DBS lead Model 3389; Medtronic, Inc.) 4 years prior to the current presentation, with significant symptomatic improvement (and relapse of her symptoms during intervals when the stimulator was turned off) (Fig. 1). Evaluation in the office revealed additional symptoms of blurry vision, dizziness, and progressively worsening headaches. She underwent an emergent CT scan of the brain without contrast enhancement, and it revealed a $3-\mathrm{cm}$ hypodense intraaxial mass within the right anterior inferior frontal lobe with moderate surrounding vasogenic edema resulting in localized sulcal effacement and mild effacement of the right frontal horn. A rounded 10-mm hypoattenuating mass was also visualized within the anterior superior left paramedian parietal lobe, associated with mild surrounding vasogenic edema. The presence of a DBS lead was also noted. The findings were concerning for metastatic cancer, and the patient was admitted for further evaluation and management. She underwent systemic imaging studies, which revealed a mass in the left lung, mediastinal lymph node conglomerates, and a left-sided pleural effusion. Pleural fluid cytology and immunohistochemistry analysis were positive for an adenocarcinoma, consistent with a primary lung tumor. An MRI study of the brain was performed, and it confirmed at least 8 intracranial rim-enhancing lesions consistent with metastatic disease. Again visualized on the MRI was the presence of a left thalamic DBS electrode and lead. Given these findings, the patient was referred for consideration for palliative whole-brain radiation therapy.

Prior to starting radiation therapy, the patient underwent simulation in the supine position with a 3-point Aquaplast mask for immobilization. At the time of her radiation therapy treatment planning, the leads of her DBS unit were contoured on each axial CT scan slice, with a plan to deliver a total dose of $30 \mathrm{~Gy}$ in 10 fractions to the whole brain using $6 \mathrm{MV}$ photons via parallel opposed lateral fields using a German helmet technique to ensure coverage of the entire cranial contents (Fig. 2).
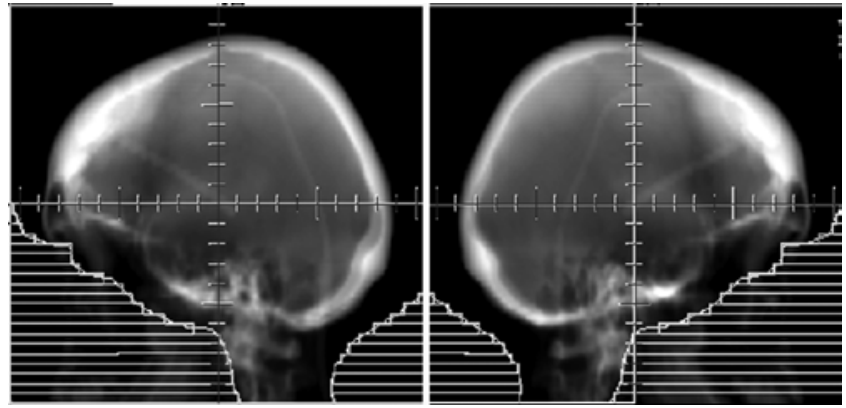

FIG. 2. Left and right lateral digitally reconstructed radiographs illustrating the whole-brain radiation therapy field with adequate blockage to ensure coverage of the entire cranial contents.

\section{Treatment and Posttreatment Course}

The mean and maximum calculated doses to the DBS electrode leads were 28 Gy and 33 Gy respectively (Fig. 3). The dose to the pulse generator itself (located in the infraclavicular region, outside of the treatment field) was measured to be $6.1 \mathrm{cGy} /$ fraction using a thermoluminescent dosimeter placed over the device during treatment. Based on a literature review, the first 3 fractions were administered with the DBS turned on, without any evidence of stimulator-related toxicity or dysfunction. Subsequently, based on a discussion with the engineers from the device manufacturer (Neuromodulation Medical Affairs, Medtronic, Inc.), the stimulator was temporarily turned off prior to and during each treatment fraction to minimize any potential for dose enhancement around the metallic leads. The patient completed her full course of $30 \mathrm{~Gy}$ in 10 fractions without any evidence of toxicity, new neurological changes, or dysfunction related to the DBS device. She was seen in follow-up 4 weeks after completion of radiation therapy and had no increase in tremor or apparent change in function of the DBS unit due to her radiation therapy, although she experienced the expected degree of treatment-related fatigue and alopecia. She ultimately died from progression of her systemic disease approximately 8.5 weeks after her first fraction of radiation therapy without any worsening of
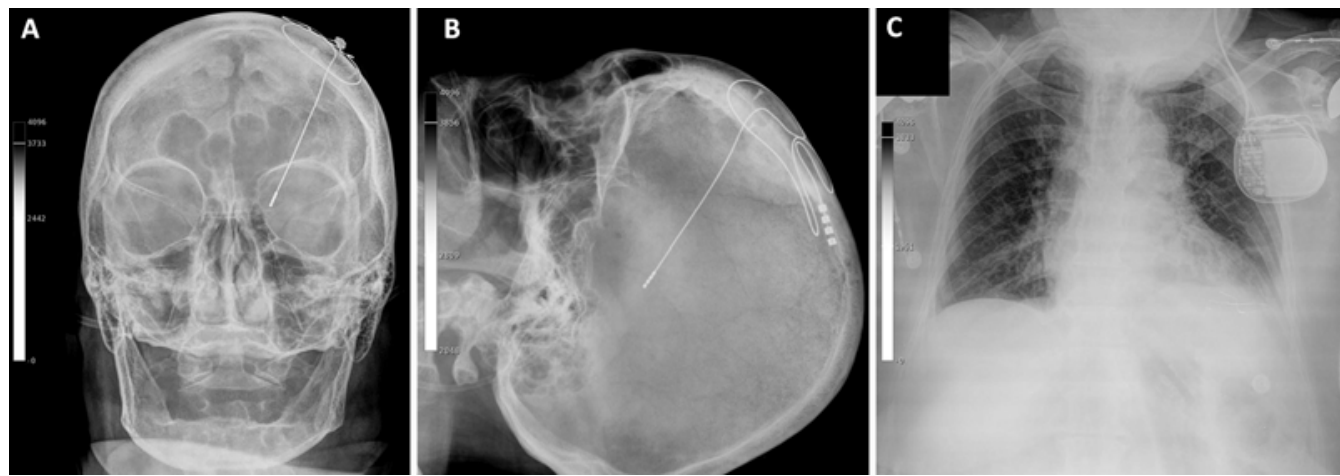

FIG. 1. A and B: Cervical anteroposterior (A) and lateral $(B)$ radiographs obtained after placement of the DBS lead (entering the left side of the cranium). C: Anteroposterior chest radiograph obtained with a portable unit demonstrating placement of a leftsided neurostimulator generator with an associated electrode lead. The stimulator leads are visualized crossing the left side of the neck and (in A and B) entering the left side of the calvaria. 


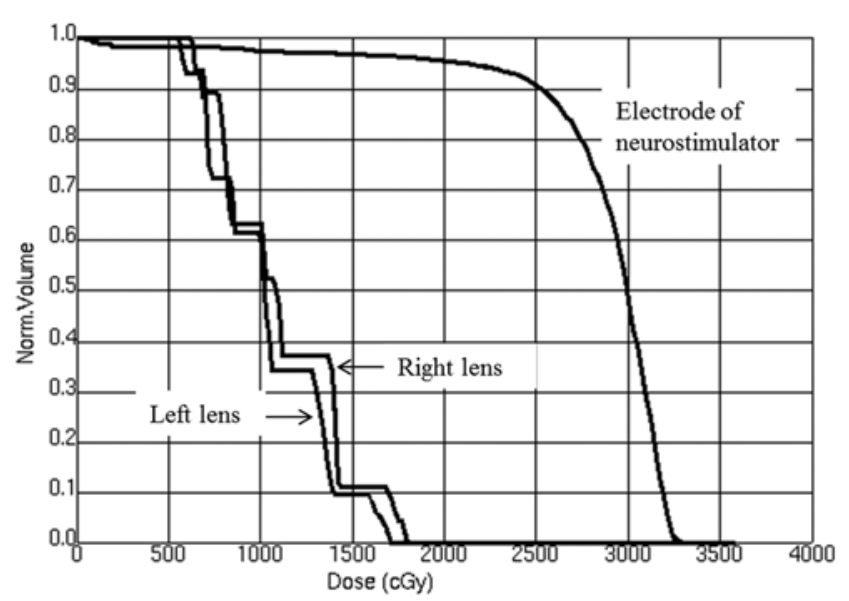

FIG. 3. A dose-volume histogram illustrating the doses to the left lens, right lens, and the intracranial lead components of the deep brain stimulator. The mean and maximum doses to the device were $28 \mathrm{~Gy}$ and 33 Gy, respectively.

her tremors or other evidence of neurological deterioration or dysfunction of the DBS device.

\section{Discussion}

Patients with dystonic movement disorders, such as essential tremors and Parkinson's disease, are initially treated with medical therapy, but $25 \%-55 \%$ of these patients may have no significant initial response to therapy or will develop medication-refractory disease..$^{11}$ Deep brain stimulation provides an excellent opportunity for symptomatic relief in eligible patients. ${ }^{2,12}$ The target most commonly used to manage essential tremor is the ventral intermedius nucleus of the thalamus on the side opposite to the desired effects. The implantable pulse generator (IPG) powers and controls the stimulation. It is typically implanted in the infraclavicular area and is connected to the electrode lead(s) via extension cables tunneled subcutaneously. Stimulation-related side effects can usually be resolved with reprogramming of the device. ${ }^{2}$ Patients with DBS devices cannot undergo MRI, with only the exception of head-only MRI studies obtained under strict guidelines provided by the manufacturer.

Concerns regarding the irradiation of medical devices, ranging from pacemakers to neuromodulators, include imaging artifacts that may distort delineation of the target volumes or identification of organs at risk, miscalculations encountered in the treatment planning system regarding dose delivery, and interference with the neurostimulation function of the device itself. ${ }^{4}$ DBS units present unique potential complications for ionizing radiation in that they not only include an IPG but also have metallic electrode leads that are implanted deep in the brain. Exposure to both sets of components can lead to separate, but clinically important risks to the patient.

Exposure of the neurostimulator device to ionizing radiation or any electromagnetic interference may result in a range of side effects from minor patient discomfort to direct tissue damage and brain injury. This may be due to a minor change in stimulation output from the pulse gen- erator, or in more serious circumstances, changes in the device memory and control circuitry (Activa Deep Brain Stimulation Brief Summary of Labeling, Medtronic, personal communication 9/24/2014). There are only 2 previous case reports detailing the safety of irradiation of a pulse generator device and 1 report on the safety of cranial radiation therapy in a patient with an implanted DBS unit. In the report by Borkenhagen and colleagues, a patient with bilateral DBS devices implanted for the treatment of Parkinson's disease underwent radiation therapy to a left upper lung tumor directly underneath the location of the IPG. ${ }^{1}$ The mean dose to the device was $5.53 \mathrm{~Gy}$, and the maximum dose was 48.12 Gy. Follow-up interrogation of the device revealed no changes in its settings or evidence of malfunctioning. In a similar report by Mazdai and colleagues, a patient being treated with DBS for severe Parkinson's disease underwent radiation therapy to the head and neck. ${ }^{13}$ In this case, the estimated dose to the device was $7.5 \mathrm{~Gy}$. In both cases, each patient's device was interrogated after radiation therapy and was found to be in good working order despite receiving a radiation dose exceeding typical pacemaker tolerances (3-5 Gy). ${ }^{14}$ In the third case, a patient who had a DBS unit implanted for the treatment of severe Parkinson's disease underwent a course of hypofractionated radiation therapy (21 Gy in 3 fractions of 7 Gy per fraction delivered over 1 week) for the treatment of 2 brain metastases using stereotactic dynamic intensitymodulated arc therapy. ${ }^{6}$ In this case the electrodes received less than $1 \mathrm{~Gy}$ and the pulse generator received less than $0.01 \mathrm{~Gy}$. Concerning our patient, the IPG was well outside the field of radiation therapy and received a nominal dose of only $6.1 \mathrm{cGy} /$ fraction (61 cGy total), but the electrodes received a maximum of $33 \mathrm{~Gy}$. Importantly, these 4 cases may not demonstrate safety of device irradiation to such high levels and are limited in direct application to other patients due to short-term follow-up or random error. For example, with regard to pacemakers and defibrillators, in a study by Gossman and colleagues, only $3 \%$ of patients had a change in functionality of their device, yet changes were observed in some patients receiving doses as low as $0.3 \mathrm{~Gy} .{ }^{5}$ It is also important to note that some neurostimulator devices have even demonstrated sensitivity to diagnostic CT scans, which are often obtained during the initial diagnostic workup and for radiation therapy treatment planning (DBS \& Radiation of Brain, Medtronic, personal communication 9/24/2014).

The general recommendation is to turn off the neurostimulator device if it is to be exposed to radiation therapy, although this should also be decided on a case-by-case basis, since this period could exacerbate the patient's symptoms for the duration of the treatment (Activa Deep Brain Stimulation Brief Summary of Labeling, Medtronic, personal communication 9/24/2014). In general, this temporary risk is likely to be small given the short treatment time necessary for radiation therapy setup and delivery and given that many patients are already instructed to turn off the device at night or even take "stimulation holidays" at weekly intervals to prevent tissue habituation. ${ }^{16}$ The greater concern related to cancer treatment in a patient with a neurostimulation device is tissue injury. If the treatment properties are altered by the device or cause the device 
to stimulate at doses above those considered safe, injury to the brain around the leads may occur. Interestingly, in the case reported by Borkenhagen and colleagues, ${ }^{1}$ it was decided that the IPG should be turned off prior to each fraction of radiation therapy. Conversely, Mazdai and colleagues ${ }^{13}$ took the approach of turning the device settings to their maximal output to minimize movement (i.e., tremor) during treatment delivery. In both of these cases, the device appeared to function correctly during the course of radiation therapy with no report of aberrant functioning or adverse effect to the patient. Based on a discussion with the treatment team, neurosurgeon, and device engineers, it was decided to turn off the neurostimulator for our patient prior to each fraction of radiation therapy delivery. Prior to this change taking effect, however, the patient did receive 3 fractions of radiation therapy with the neurostimulator left on without any significant adverse effect.

Preclinical modeling studies have demonstrated that other implanted medical devices, such as cochlear implants, can be safely irradiated to therapeutic doses $(<80$ Gy) using conventionally fractionated and hyperfractionated treatment schedules. ${ }^{10}$ However, to the best of our knowledge, there are no reports describing the safety of whole-brain radiation therapy in patients with implanted deep brain stimulators. As previously detailed, DBS devices contain long electrically insulated leads that are able to transmit electrical pulses along their entire length toward the targeted area. ${ }^{2}$ Therefore, the electrode lead and other components could act as conductive pathway and lead to tissue damage and brain injury even with the IPG out of the field of radiation. Moreover, exposure to ionizing radiation therapy can also disrupt the electrical circuit. ${ }^{9}$ Although this has never been demonstrated in the case of therapeutic radiation therapy delivery, the effect of electromagnetic interference on neurostimulator function has been demonstrated in diagnostic imaging and diathermy treatments. For example, a study by Gorny and colleagues demonstrated that 3.0-T MRI sequences can result in local temperature elevations at DBS electrodes. ${ }^{3}$ In their study, multiple measurements were performed both with DBS at the normal stimulation rate and with the output zeroed and the IPG turned off. During one of the MRI pulse sequences, they observed a $26 \%$ increase in temperature elevations for the runs performed with the IPG turned on, but this was not demonstrated on other pulse sequences. Moreover, during runs performed in certain MRI pulse sequences, they observed interruption in the function of the IPG if it was left on during the scan. Henderson and colleagues reported a case of permanent neurological injury produced by heating of a DBS electrode in a patient with Parkinson's disease who underwent an MRI. ${ }^{7}$ Nutt and colleagues reported on a patient with bilateral implanted neurostimulators who sustained significant brain injury and died as a result of excessive tissue heating from diathermy treatments (with DBS in operation mode during the diathermy treatments). $)^{15,17}$

One of the methods used to reduce radiation exposure to the device is to place a lead shield over the pulse generator during treatment. We feel that this should be considered for patients who have limited brain metastases and no evidence of disease near the site of the pulse generator. On the other hand, if areas near the generator are involved, or if disease is significantly multifocal (i.e., more than 5 metastases), blocking the site of the pulse generator may be associated with high risk of disease recurrence, in which case we recommend discussing the risks and benefits with the patient and proceeding with treatment.

\section{Conclusions}

As the indications for implantation of neuromodulatory medical devices continue to increase, it is important to document clinical experience in patients receiving radiation therapy. Regarding patients receiving whole-brain radiation therapy or radiation therapy for head and neck malignancies, 2 types of devices encountered in clinical practice-DBS and cochlear implants-need to be considered. When encountering such circumstances, basic radiation therapy treatment principles include: modifying beam entry angles to avoid direct beam entry through a device, accurately measuring and documenting the maximum dose delivered to the device, and contacting the manufacturer to ensure that the radiation tolerance of the device is not exceeded. Neurosurgeons and radiation oncologists should have discussions with patients about the risks of brain injury, device malfunction or failure of the device, and plans for rigorous testing of the device before and after radiation therapy. In these cases, multidisciplinary discussions are important to providing safe, yet effective care for these patients.

\section{References}

1. Borkenhagen JF, Morris ZS, Hoberg JR, Kozak KR, Shapiro LQ: Delivery of definitive dose external beam radiation in close proximity to an implanted deep brain stimulator. Pract Radiat Oncol 4:294-297, 2014

2. Flora ED, Perera CL, Cameron AL, Maddern GJ: Deep brain stimulation for essential tremor: a systematic review. Mov Disord 25:1550-1559, 2010

3. Gorny KR, Presti MF, Goerss SJ, Hwang SC, Jang DP, Kim I, et al: Measurements of RF heating during 3.0-T MRI of a pig implanted with deep brain stimulator. Magn Reson Imaging 31:783-788, 2013

4. Gossman MS, Paralikar KJ, Hebb AO, Wilkinson JD, Graves-Calhoun AR, Lawson RC, et al: Effects on radiation oncology treatments involving various neuromodulation devices. J XRay Sci Technol 19:443-456, 2011

5. Gossman MS, Wilkinson JD, Mallick A: Treatment approach, delivery, and follow-up evaluation for cardiac rhythm disease management patients receiving radiation therapy: retrospective physician surveys including chart reviews at numerous centers. Med Dosim 39:320-324, 2014

6. Guy JB, Levy A, Malkoun N, Chargari C, Bigot L, Magné N: Preventing radiotherapy-induced side effects on deep brain stimulators: the need for a multidisciplinary management. $\mathbf{B r}$ J Neurosurg 28:107-109, 2014

7. Henderson JM, Tkach J, Phillips M, Baker K, Shellock FG, Rezai AR: Permanent neurological deficit related to magnetic resonance imaging in a patient with implanted deep brain stimulation electrodes for Parkinson's disease: case report. Neurosurgery 57:E1063, 2005

8. Hurkmans CW, Knegjens JL, Oei BS, Maas AJ, Uiterwaal GJ, van der Borden AJ, et al: Management of radiation oncology patients with a pacemaker or ICD: a new comprehensive practical guideline in The Netherlands. Radiat Oncol 7:198, 2012 
9. Kawamura S, Ono S, Kuga N, Shiba T, Fujimoto H, Toyoshima T, et al: Effects of irradiation on the components of implantable pacemakers. Igaku Butsuri 23:73-80, 2003

10. Klenzner T, Knapp F, Röhner F, von Wallenberg E, Mauch H, Pedersen $\mathrm{P}$, et al: Influence of ionizing radiation on nucleus 24 cochlear implants. Otol Neurotol 26:661-667, 2005

11. Louis ED: Clinical practice. Essential tremor. N Engl J Med 345:887-891, 2001

12. Machado A, Rezai AR, Kopell BH, Gross RE, Sharan AD, Benabid AL: Deep brain stimulation for Parkinson's disease: surgical technique and perioperative management. Mov Disord 21 (Suppl 14):S247-S258, 2006

13. Mazdai G, Stewart DP, Hounsell AR: Radical radiation therapy in a patient with head and neck cancer and severe Parkinson's disease. Clin Oncol (R Coll Radiol) 18:82-84, 2006

14. Munshi A, Agarwal JP, Pandey KC: Cancer patients with cardiac pacemakers needing radiation treatment: a systematic review. J Cancer Res Ther 9:193-198, 2013

15. Nutt JG, Anderson VC, Peacock JH, Hammerstad JP, Burchiel KJ: DBS and diathermy interaction induces severe CNS damage. Neurology 56:1384-1386, 2001

16. Plaha P, Patel NK, Gill SS: Stimulation of the subthalamic region for essential tremor. J Neurosurg 101:48-54, 2004

17. Ruggera PS, Witters DM, von Maltzahn G, Bassen HI: In vitro assessment of tissue heating near metallic medical implants by exposure to pulsed radio frequency diathermy. Phys Med Biol 48:2919-2928, 2003

\section{Disclosure}

Dr. Machado reports an ownership interest in Autonomic Technologies, Inc., Cardionomics, and Enspire and a consultant relationship with Functional Neuromodulation and Spinal Modulation. Dr. Suh reports a consultant relationship with Varian Medical Systems. Dr. Chao reports being a member of the speakers' bureau for Varian Medical Systems (honorarium).

\section{Author Contributions}

Conception and design: Stephans, Kotecha, Berriochoa, Machado. Acquisition of data: Stephans, Kotecha, Berriochoa. Analysis and interpretation of data: Stephans, Kotecha, Berriochoa, Murphy, Chao, Suh. Drafting the article: Stephans, Kotecha. Critically revising the article: all authors. Reviewed submitted version of manuscript: all authors.

\section{Correspondence}

Kevin L. Stephans, Department of Radiation Oncology, Taussig Cancer Institute, 9500 Euclid Ave., T28, Cleveland, OH 44195. email: stephak@ccf.org. 